\title{
DISCRETE TIME ANALYSIS OF A SLOTTED TRANSMISSION SYSTEM
}

\author{
Bart VAN AREM* \\ Faculty of Applied Mathematics, University of Twente, 7500 AE Enschede, The Netherlands
}

(Received September 1987; revised August 1988)

\begin{abstract}
Scope and Purpose-In many transmission systems a number of transmission channels or slots are available for packets containing digital information. These packets are generated by a number of sources alternating between active and passive periods, slowly compared to the transmission process. When more packets are generated than the system can handle, overflowing packets are stored in a buffer with infinite capacity. From the point of view of performance analysis of the transmission system it is of interest to know the expected number of packets in the buffer. This motivates the present study of a buffer system with a correlated input process. We give not only a theoretical analysis, but also describe a numerical implementation. Analytical results for applications are compared with simulation results. It appears that within a certain parameter range the analytical results are calculated faster.
\end{abstract}

\begin{abstract}
This paper concerns the performance analysis of a slotted transmission system. Packets of equal size arrive at the transmission facility which can handle a certain maximum number of packets per time-unit called frame. Transmission is assumed to be gated at the start of frames. Temporary overflow is stored in a buffer with infinite capacity. The packet arrival process is described by a Markov chain with finite state space. We derive the stationary expected number of packets in the buffer and the stationary expected packet delay. We also formulate and describe the implementation of an algorithm to compute these quantities. The accuracy of the algorithm is checked by simulation. A realistic traffic model is given and specific parameters are chosen. Results and numerical aspects are evaluated.
\end{abstract}

\section{INTRODUCTION}

In the design and analysis of communication systems more and more use is made of stochastic performance evaluation. It is not surprising that this field of research has received much attention during the past several years. New communication systems are being developed so new models have to be made to analyse the performance of these systems. In particular much research has been done on integrated voice/data communication systems. In most analyses a synchronous time division multiplexed (TDM) frame structure is investigated: time is divided into frames of equal, fixed size. In each frame certain parts are dedicated to voice and data traffic respectively. Although future developments also indicate other ways of integration of different traffic types, e.g. by using asynchronous schemes (ATDM), there are certain types of traffic that will still require synchronous transmission.

In our model we assume a synchronous transmission scheme. We consider a finite number of sources that can be active or passive. When a source is active it produces one packet every frame. All packets have equal size. In each frame a fixed number of equally sized slots, adapted to thc packet size is available. When more packets are produced than the number of slots available, the system is said to be in overload mode and the overflowing packets are stored in a buffer with infinite capacity. We assume that packets generated during a particular frame can only be transmitted in subsequent frames.

This model can be applied to many situations. Consider the situation in which a fixed number of slots in each frame is available for voice traffic. The other slots in each frame can be used by data traffic. Suppose we have a finite number of voice sources on which digital speech interpolation (DSI) is performed, so that only active, speaking sources produce a digital packet. Now assume that temporary overflow of voice packets can be stored in a buffer, then performance measures for speech quality can be calculated with the model in this paper.

* Bart van Arem received the M.S. degree in Applied Mathematics in 1986 from the University of Twente, Enschede, The Netherlands. Since 1986 he has been with the University of Twente, where he is doing his $\mathrm{Ph} . \mathrm{D}$. research on stochastic performance analysis of slotted communication systems. His research interests include queueing theory, performance evaluation of computer and communication models and traffic theory. 
It is not necessary, however, to restrict our applications to one particular kind of traffic. When we dedicate different frame sections to different traffic types, then by the lack of interaction we can analyse all parts independently. More generally, the model can be applied to any situation where a number of sources can be active or passive. When active the source periodically claims a fixed part of the service capacity available for all sources. When the requested service can not immediately be granted, it is postponed and granted later.

This paper has been extracted from two reports $[1,2]$ in which a more detailed analysis is given. On request these reports can be obtained from the author. In this paper it is assumed that the reader has some knowledge of stochastic modelling and the analysis of functions of one complex variable. This background mainly applies to Section 4 where a theoretical analysis is presented.

The organisation of this paper is as follows. In Section 2 we give the description of the model under consideration. After that we discuss related studies in Section 3 and a theoretical analysis is presented in Section 4. In Section 5 we give an algorithm and comment on the implementation and validation of this algorithm. In Section 6 we introduce a specific traffic model and choose realistic parameters. In Section 7 results for this model are given and in Section 8 numerical aspects of the algorithm are discussed. Finally in Section 9 we evaluate the results and draw some conclusions.

\section{MODEL DESCRIPTION}

We consider a number of $N$ sources that alternate between active and passive periods. Time is divided into frames of duration $f$. For each source at most one transition between active and passive status can take place during a frame. The number of packets produced in a frame is equal to the number of active sources in that frame. A source is considered passive during a frame only if it was passive during the entire frame. We let $T_{n}$ be the number of packets generated in frame $n$ and assume that $\left(T_{n}\right), n=0,1,2, \ldots$ is an irreducible and aperiodic Markov chain with state space $S=\{0,1, \ldots, N\}$ and $(N+1) \times(N+1)$ stationary probability matrix $P=\left(p_{i j}\right)$. The arrival times of packets during a frame are independent random variables which are identically and uniformly distributed over that frame.

During each frame a maximum number of $R(R \leqslant N)$ packets can be transmitted. Transmission is assumed to be gated at starts of frames. Thus packets arriving during frame $(n-1)$ can not be transmitted earlier than in frame $n$. Packets waiting for transmission are stored in a buffer with infinite capacity. Denote by $B_{n}, n=0,1, \ldots$ the number of packets in the buffer just before the end of frame $n$. Obviously $\left(B_{n}, T_{n}\right), n=0,1,2, \ldots$ is a two-dimensional irreducible, aperiodic Markov chain with state space $\{0,1,2, \ldots\} \times S$ and system evolution described by (see also Fig. 1)

$$
B_{n+1}=\left[B_{n}-R\right]^{+}+T_{n+1}, \quad n=0,1,2, \ldots
$$

where $[x]^{+}=\max (0, x)$. Because we are interested in stationary behaviour and stability requirements will be assumed satisfied the distribution of $\left(B_{0}, T_{0}\right)$ can be chosen arbitrarily.

Packets arriving in different frames are transmitted according to a first-in first-out service discipline (FIFO). Packets arriving in the same frame are transmitted in random order. The delay is defined as the difference between the time at the start of the frame in which the packet is transmitted and the arrival time of the packet.

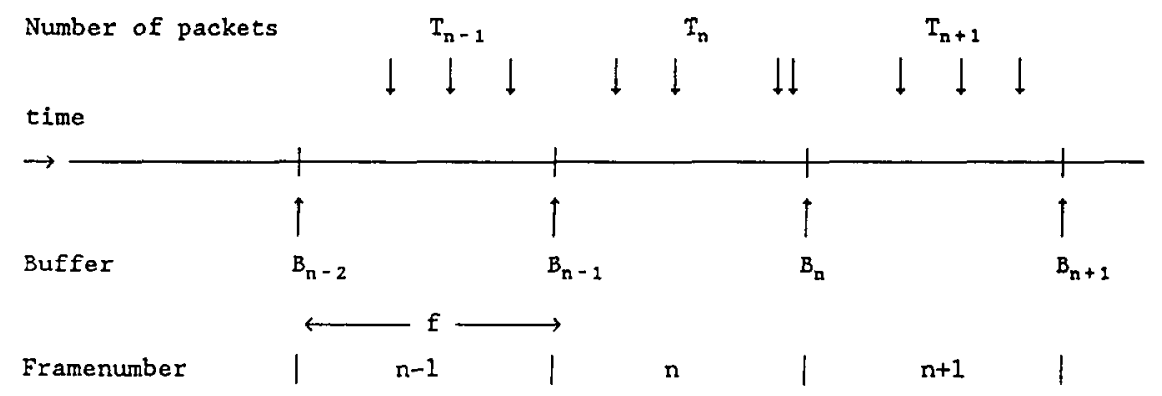

Fig. 1. System evolution 
We shall be interested in the stationary behaviour of the system. Because $T_{n}$ is irreducible and aperiodic, $T_{n}$ converges weakly to a random variable $T$ with $E T<\infty$ when $n \rightarrow \infty$. To guarantee stability we shall impose the following condition on the system:

\section{Condition 1: $E T<R$}

It can be shown by a regenerative argument that when condition 1 is satisfied, $\left(B_{n}, T_{n}\right)$ converges weakly to a pair of random variables $(B, T)$ with $E B<\infty$ when $n \rightarrow \infty$.

In the theoretical analysis in this paper, our first task shall be to derive the probability generating function of $B$. Once this function is available we shall be able to derive quantities such as $E B$ and the stationary expected packet delay.

\section{RELATED STUDIES}

The literature on stochastic performance analysis of communication systems is very extensive. There are many studies of models related to our model. It is not our purpose to review all work in this area, but we will mention studies from three research orientations that are directly related to our model.

The first orientation concerns computer communication models. In this field in [3] a similar model to ours is analysed in the single channel case $(R=1)$ with an infinite user population $(N=\infty)$. This means that $T_{n}$ can take values in $\{0,1,2, \ldots\}$ and has no finite upper bound.

The second orientation originates from packetized voice communication systems and integrated voice/data communication systems. In [4] a packetized voice communication system in continuous time is analysed that is also similar to our model. An embedded Markov chain is considered at two types of time instants: firstly at instants of a change in the number of active sources and secondly at instants of queue length increase or decrease. The queue length distribution is derived using Neuts' matrix-gcometric method. Many related studies consider a movable boundary transmission scheme. The number of slots available for data traffic is regulated by the number of (active) voice calls. In these models only data traffic can be stored in a buffer. The content of this buffer is the random variable of interest. In [5] the movable boundary scheme is studied in discrete time. Batches of data packets arrive according to a Poisson process. Batch sizes have an arbitrary distribution. Overflowing data packets can be stored in a buffer with infinite capacity. In the analysis of the buffer content techniques are employed that resemble the techniques in this paper.

As a third orientation we mention a particular development from the second: approximations using fluid-flow models for data traffic. Fluid reservoirs with Markov regulated input and/or output were studied in [6-8] and more recently in [9]. In [10] Wiener-Hopf factorization techniques are employed which appear to be very powerful.

\section{THEORETICAL ANALYSIS}

In this section we shall give a theoretical analysis of the model described in Section 2. It is not our purpose to go into mathematical details, for which the reader is referred to [1]. To give a brief introduction to the theoretical analysis we will first globally describe the typical procedure employed to solve the present model.

Like in many stochastic analyses a transformation is performed from probability distributions of the quantity of interest to functions of a complex variable. In our case it can be proved that these functions must be analytic inside the unit circle and continuous on the boundary. By relating $B_{n+1}$ and $B_{n}$ we can find a relation between the corresponding complex functions, which is still time-dependent because of the presence of the parameter $n$. Assuming that $n \rightarrow \infty$ the two functions converge to the same limiting function and we find an equation describing the stationary behaviour of the system. From this equation an expression for the solution follows. However, three problems are now encountered. Firstly a number of unknowns still occur in the solution, secondly the solution is not analytic inside the unit circle and continuous on the boundary and thirdly the solution does not yet correspond to a proper probability distribution in the sense that it should be normalised. It appears that the unknowns can be determined uniquely in such a way that the second and third problem are solved. Finally the solution allows us to obtain system characteristics. 
The analysis in this paper falls apart into three steps: firstly we derive a characteristic equation, secondly we show how this equation can be solved and thirdly we finish the job by indicating how the expected queue length and expected packet delay can be calculated from this solution.

Step 1. Derivation of characteristic equation

In Section 2 we introduced $[x]^{+}=\max (0, x)$. We also denote $[x]^{-}=\min (0, x)$ and we will use the identity

$$
z^{[x]^{+}}=z^{x}+1-z^{[x]^{-}} .
$$

Let $I(\mathrm{~A})$ denote the indicator function of the event A. Define $J_{l}^{(n)}(z) \equiv E\left[z^{B_{n}} I\left(T_{n}=l\right)\right],|z| \leqslant 1, l \in S$, then from (1) we obtain

$$
\begin{aligned}
J_{l}^{(n+1)}(z) & =E\left[z^{B_{n}-R+l} I\left(T_{n+1}=l\right)\right]+E\left[z^{l}\left(1-z^{\left[B_{n}-R\right]^{-}}\right) I\left(T_{n+1}=l\right)\right] \\
& =z^{l-R} \sum_{j \in S} p_{j l} J_{j}^{(n)}(z)+z^{l} \sum_{j \in S} p_{j l} \sum_{i=0}^{R-1}\left(1-z^{i-R}\right) P\left(B_{n}=i, T_{n}=j\right), \quad|z| \leqslant 1, l \in S .
\end{aligned}
$$

Now let

$$
\begin{gathered}
J_{l}(z) \equiv \lim _{n \rightarrow \infty} J_{l}^{(n)}(z), \quad|z| \leqslant 1, l \in S, \\
P(B=i, T=j) \equiv \lim _{n \rightarrow \infty} P\left(B_{n}=i, T_{n}=j\right) .
\end{gathered}
$$

It can be shown [1] that these limits exist when condition 1 is satisfied. By letting $n \rightarrow \infty$ equation (3) converges to

$$
J_{l}(z)=z^{l-R} \sum_{j \in S} p_{j l} J_{j}(z)+z^{l} \sum_{j \in S} p_{j l} \sum_{i=0}^{R-1}\left(1-z^{i-R}\right) P(B=i, T=j), \quad|z| \leqslant 1, l \in S .
$$

By introducing vector-matrix notation we can write (4) in a more convenient form. Let

$$
J(z)=\left[J_{0}(z), \ldots, J_{N}(z)\right]
$$

$\alpha(z)=\left[\alpha_{0}(z), \ldots, \alpha_{N}(z)\right]$,

$$
\begin{aligned}
& \text { where } \begin{array}{c}
\alpha_{l}(z)=z^{R} \sum_{j \in S} z^{l} p_{j l} \sum_{i=0}^{R-1}\left(1-z^{i-R}\right) P(B=i, T=j), l \in S \\
A(z)=\operatorname{diag}\left[1, z, \ldots, z^{N}\right]
\end{array}
\end{aligned}
$$

and let $I$ denote the $(N+1) \times(N+1)$ identity matrix. Equation (4) can now be written as

$$
J(z)\left[z^{R} I-P A(z)\right]=\alpha(z), \quad|z| \leqslant 1
$$

which is the characteristic equation we were looking for.

Step 2. Solution of characteristic equation

We want to solve $J(z)$ from (5). However, $\alpha(z)$ contains the unknowns

$$
P(B=i, T=j), \quad i=0, \ldots, R-1 ; j \in S .
$$

But from (1) it follows that $P(B=i, T=j)=0$, when $i<j$, so that exactly $\frac{1}{2} R(R+1)$ unknowns remain. Consider the zeros of $\operatorname{det}\left[z^{R} I-P A(z)\right]$. These zeros become zeros in the denominator of any coordinate of the vector

$$
J(z)=\alpha(z)\left[z^{R} I-P A(z)\right]^{-1} .
$$

However, because $J(z)$ is a vector of partial generating functions, each coordinate must be complex analytic on $|z|<1$ and bounded on $|z| \leqslant 1$. This implies that the zeros of $\operatorname{det}\left[z^{R} I-P A(z)\right]$ in $|z| \leqslant 1$ must also be zeros of the numerator of any coordinate of (6). 
Before making a statement about the zeros of $\operatorname{det}\left[z^{\mathrm{R}} I-P A(z)\right]$ in $|z| \leqslant 1$ we need a second condition to be satisfied.

Let

$$
L=\left[\begin{array}{ccccc}
-p_{00} & -p_{01} & \cdots & -p_{0, R-1} & -p_{0, R} \\
-p_{10} & -p_{11} & \ldots & -p_{1, R-1} & -p_{1, R} \\
\vdots & \vdots & & \vdots & \vdots \\
-\dot{p_{R-1,0}} & -p_{R-1,1} & \cdots & -p_{R-1, R-1} & -p_{R-1, R} \\
-p_{R, 0} & -p_{R, 1} & \cdots & -p_{R, R-1} & 1-p_{R, R}
\end{array}\right] .
$$

Condition 2. $\operatorname{det} L=\neq 0$

It can be shown [1] that provided conditions 1 and 2 are satisfied $\operatorname{det}\left[z^{R} I-P A(z)\right]$ has:

a zero of multiplicity 1 in $z=1$

a zero of multiplicity $\frac{1}{2} R(R+1)+(N-R) R$ in $z=0$

$\frac{1}{2} R(R+1)-1$ zeros in $0<|z|<1$.

The second condition ensures [1] that the multiplicity of the zero in $z=0$ is exactly $\frac{1}{2} R(R+1)+$ $(N-R) R$. If it is violated, then $z=0$ has a larger multiplicity and the present procedure can no longer be applied. Although the procedure might be extended to cover cases for which condition 2 is violated and although it is indeed possible to construct such cases [1], we did not encounter violations of condition 2 in the realistic cases we considered. In Section 8 we will discuss this matter further.

It appears [1] that the zeros in $z=0$ and $z=1$ are always cancelled by zeros in the numerator of any coordinate of $(6)$. Furthermore we can determine $\frac{1}{2} R(R+1)-1$ unknowns such that also the zeros of the denominator on $0<|z|<1$ are cancelled. The final unknown can be determined by a normalisation condition, because we should have: $\sum_{i \in S} J_{i}(1)=1$. It can easily be verified that this procedure gives rise to a set of $\frac{1}{2} R(R+1)$ linear simultaneous equations. Now we have to impose the following restriction:

Condition 3: The set of linear simultaneous equations to derive the unknowns is independent

It can be shown [1] that it does not make any difference which coordinate is used to obtain the unknowns, because these are uniquely determined probabilities.

Step 3: Derivation expected queue length and delay

Denote by $v$ the stationary distribution vector $\left[v_{0}, v_{1}, \ldots, v_{N}\right]$ of $T$ obtained from

$$
v P=v, \quad \sum_{i=0}^{N} v_{i}=1 .
$$

The expected stationary number of packets generated in one frame, $E T$, can now be calculated as $E T=\sum_{i=1}^{N} i v_{i}$.

Denote the probability generating function of $B$ by $X(z)$, then we can derive:

$$
\begin{aligned}
X(z) & =E\left[z^{B}\right] \\
& =\sum_{i=0}^{N} E\left[z^{B} I(T=i)\right] \\
& =\sum_{i=0}^{N} J_{i}(z) .
\end{aligned}
$$

Now $E B$ follows from

$$
E B=X^{\prime}(1)
$$

and if $D$ is the stationary packet delay expressed in seconds, then we can find $E D$ using Little's formula as follows: clearly the expected number of packets in the buffer just before the end of a frame is $E B$ and just after the beginning of a frame $E B-E T$ (recall that the gate opens and closes at the beginning of a frame). Since the packets are assumed to arrive uniformly over a frame the 
expected number of packets in the buffer in continuous time is $E B-E T / 2$. Because the arrival intensity is equal to $E T / f$, Little's formula finally yields

$$
E D=\frac{f}{E T}\left(E B-\frac{E T}{2}\right)
$$

\section{ALGORITHM, IMPLEMENTATION AND VALIDATION}

We summarize the theoretical analysis by formulating an algorithm to obtain $E B$ and $E D$. After that we make some remarks on the implementation of the algorithm. Finally we discuss a method to validate our results.

Step 0: check condition 1 .

Step 1: calculate $\operatorname{det}\left[z^{R} I-P A(z)\right]$.

Step 2: calculate the zeros of $\operatorname{det}\left[z^{R} I-P A(z)\right]$. Check condition 2 .

Step 3: calculate $J(z)$. In this expression the unknowns

$$
P(B=i, T=j) ; \quad j=0, \ldots, R-1 ; \quad i=j, \ldots, R-1
$$

still occur in the numerator. Use the zeros from step 2 to construct a set of linear simultaneous equations to determine the unknowns.

Step 4: solve the set of linear simultaneous equations. This can be done when condition 3 is satisfied.

Step 5: recalculate $J(z)$. This is done straightforwardly by cancelling common factors in denominators and numerators.

Step 6: calculate $X(z)$ using (8).

Step 7: compute $E B$ and $E D$ from (9) and (10).

A computer program in PASCAL was written and executed on a VAX 8650 computer. All calculations were performed in double precision. Polynomials were represented using a pointer string type definition. All necessary arithmetic operators for polynomials were provided like summation, multiplication, evaluation, differentiation, division.

As a result of this environment step 1, 3, 5, 6 and 7 can easily be performed. It will be argued in Section 6 that a tri-diagonal matrix $P$ is realistic for our applications. In this case the computations become even simpler because we can use iterative schemes in the calculation of determinants. For the more complicated computations in steps 2 and 4 library routines were employed from the NAG FORTRAN numerical library. We used routine CO2AEF in step 2 and routines FO3AFF and FO4AHF in step 4.

Because of the amount of computation involved in our program it was necessary to check the correctness and accuracy of the results. This was done by stochastic simulation. A simulation program was written in PASCAL and executed in double precision on a VAX 8650 computer. The program simulates precisely the same model that is also analysed by exact calculation.

We used the regenerative method, for which we refer to [11]. In this method we use the fact that the process $\left(B_{n}, T_{n}\right)$ starts all over again every time it enters a particular fixed so-called regeneration point. The choice of the regeneration point is arbitrary because $\left(B_{n}, T_{n}\right)$ is aperiodic and irreducible. However it is important to choose these regeneration points such that sufficiently many regeneration cycles are simulated. We chose our regeneration points in $(R, R)$ which performed satisfactorily.

In [11] and [12] it is described how estimators and confidence intervals for $E B$ can be calculated from the data obtained from a sufficiently great number of regeneration cycles. In the procedure described the estimator for $E B$ is assumed to have an approximate $N\left(E B, \sigma^{2}\right)$ distribution. We used the procedure from [11] and [12] to generate a $95 \%$ confidence interval for $E B$. Unfortunately in this procedure square roots of negative values may occur, so it does not guarantee a $95 \%$ confidence interval. In this case the simulation was split up into a number of sufficiently large 
subruns. From each (independent) subrun an estimator for $E B$ is obtained. This constitutes a sample of a random variable with an approximate $N\left(E B, \sigma^{2}\right)$ distribution. A $95 \%$ confidence interval for $E B$ is easy to derive. This alternative procedure was checked by comparing it with the original procedure in the cases where the original did not fail. The alternative procedure was found to perform satisfactorily for subruns of sufficient length.

\section{TRAFFIC MODEL AND PARAMETER CHOICE}

For application of our model it is important to give a good description of the behaviour of the sources. This means that we have to specify the transition probability matrix $P$ for realistic cases. In this section we argue that we can use a small number of typical parameters to specify a tri-diagonal matrix $\boldsymbol{P}$ with a special structure. After that we shall motivate the choice of two parameter sets.

Let us first consider a single source alternating between active and passive periods. Active periods are independent identically distributed (i.i.d.) random variables with expectation $E A$ seconds. Passive periods are also i.i.d. random variables with expectation $E P$ seconds. We assume that active and passive periods are independent and that the length of an active (passive) period expressed in frames is geometrically distributed with parameter $p^{(1)}=f / E A\left(p^{(2)}=f / E P\right)$. That is for an active period $A: P(A=k)=p^{(1)}\left(1-p^{(1)}\right)^{k-1}, k \geqslant 1$. This specifies the behaviour of a single source of which we have assumed (Section 2) that if it is active only during a part of a frame, it generates a packet and is assumed to be active in that frame. This will not cause great errors because for almost all applications small values of $p^{(1)}$ and $p^{(2)}$ are realistic.

Now consider $N$ identical sources alternating between active and passive status independently. The probability that an active (passive) source becomes passive (active) during a certain frame is equal to $p^{(1)}\left(p^{(2)}\right)$. This is sufficient to obtain the transition probabilities $p_{k, l}, k, l \in S$ as defined in Section 3. Although it is possible to calculate these probabilities, they would become unnecessarily complicated. They can be simplified considerably by assuming each term $\left(p^{(1)}\right)^{2},\left(p^{(2)}\right)^{2}$ or $p^{(1)} p^{(2)}$ equal to zero. This corresponds with the assumption that state changes of more than one sources during a certain frame do not occur. Again this is realistic because $p^{(1)}$ and $p^{(2)}$ are small. Therefore we find:

$$
p_{k, l}= \begin{cases}k p^{(1)} & l=k-1, l>0 \\ 1-k p^{(1)}-(N-k) p^{(2)} & l=k \\ (N-k) p^{(2)} & l=k+1, l<N \\ 0 & \text { otherwise. }\end{cases}
$$

Evidently we have to see to it that all probabilities are nonnegative, but since $p^{(1)}$ and $p^{(2)}$ are small and $N$ will not be very large in our applications this condition will in general be satisfied.

This gives us the following transition probability matrix:

$$
P=\left[\begin{array}{cccccc}
1-N p^{(2)} & N p^{(2)} & & 0 & \cdots & 0 \\
p^{(1)} & 1-p^{(1)}-(N-1) p^{(2)} & & (N-1) p^{(2)} & & \vdots \\
0 & 2 p^{(1)} & \ddots & \ddots & & \vdots \\
\vdots & \ddots & \ddots & \ddots & \ddots & 0 \\
\vdots & \ddots & \ddots & \ddots & \ddots & p^{(2)} \\
0 & 0 & \cdots & 0 & N p^{(1)} & 1-N p^{(1)}
\end{array}\right]
$$

Summarizing the model we give a procedure to estimate $P$ :
1. Specify $f$ and $N$
2. Estimate $E A$ and $E P$
3. Calculate $p^{(1)}=f /(E A)$
$p^{(2)}=f /(E P)$

4. Calculate $P$ using (11). 
In this section we also choose two parameter sets that specify the matrix $P$ by the traffic model described in the previous section. The first model is inspired by real applications and the second model is chosen to show that the realistic parameters impose severe numerical restrictions. In all applications we take a frame length $f=0.01$ seconds. This is a typical value for, e.g. a local area network. Estimates for $E A$ and $E P$ will typically depend on the application. We will let the value of the number of sources $N$ depend on numerical experience: too great values for $N$ combined with very small elements in $P$ will appear to introduce numerical inaccuracy. We shall let $R$ vary over all values for which condition 1 is satisfied.

\section{One-way voice traffic}

Active and passive periods are interpreted as talkspurts and silent periods. Estimates for $E A$ and $E P$ depend on the language of conversation and the threshold level for distinguishing active and passive periods [13]. In literature different estimates are used that all have in common that $E A / E P=1 / 2$. This implies that one speaker is active one third of the time. Some examples of estimates are:

$$
\begin{aligned}
& (0.35,0.65):[4] \\
& (0.9,1.7):[13] \text { Table } 3 .
\end{aligned}
$$

Because the latter example is calculated from a large sample, we will adopt cstimates of [13]. Wc will consider $N=6$ conversations which was the maximum attainable, because for $N>6$ we already experienced numerical inaccuracy.

\section{Nice traffic}

It will be clear that numerical difficulties will be avoided as long as $p^{(1)}$ and $p^{(2)}$ are not too small and do not cause very small transition probabilities introducing numerical inaccuracy into the computations. However we must take care that $p^{(1)}$ and $p^{(2)}$ are not too large for application of the traffic model. This will also depend on $N$. To illustrate that it is possible to analyse more sources than with one way voice traffic, we chose $N=10$ and $E A=1 / 5$ and $E P=1 / 3$, which implies $p^{(1)}=0.05$ and $p^{(2)}=0.03$.

\section{RESULTS}

We present the results obtained with our analytical and simulation program. The case $N=R$ is added to the analytical results because it is easy to see that in this case $E B=E T$ and $E D=\frac{1}{2} f$. The results will be discussed in Sections 8 and 9. We let $\rho=E T / R$. ET was calculated straightforwardly as indicated in Section 4, step 3 .

One way voice traffic:

$$
N=6, \quad f=0.01, \quad E A=0.9, \quad E P=1.7, \quad E T=2.0823 .
$$

Table 1. Analytical results one way voice traffic

\begin{tabular}{ccccc}
\hline$R$ & $\rho$ & $\begin{array}{c}E B \\
\text { (packets) }\end{array}$ & $\begin{array}{c}E D \\
\text { (seconds) }\end{array}$ & $\begin{array}{c}\text { CPU-time } \\
\text { (seconds) }\end{array}$ \\
\hline 3 & 0.6941 & 27.5546 & 0.1273 & 0.60 \\
4 & 0.5206 & 3.9834 & 0.0141 & 0.70 \\
5 & 0.4165 & 2.1722 & 0.0054 & 1.07 \\
6 & 0.3471 & 2.0823 & 0.0050 & - \\
\hline
\end{tabular}

Table 2. Simulation results one way voice traffic

\begin{tabular}{ccccc}
\hline$R$ & $\begin{array}{c}95 \% \text { Confidence interval } \\
\text { for } E B\end{array}$ & $\begin{array}{c}\text { Interval } \\
\text { width }\end{array}$ & $\begin{array}{c}\text { Simulation time } \\
\text { (frames) }\end{array}$ & $\begin{array}{c}\text { CPU-time } \\
\text { (seconds) }\end{array}$ \\
\hline 3 & $26.7121-27.5155$ & 0.8033 & $4 \cdot 10^{7}$ & 648.75 \\
4 & 3.82964 .0157 & 0.1861 & $64 \cdot 10^{5}$ & 97.12 \\
5 & $2.0895-2.2070$ & 0.1175 & $4 \cdot 10^{5}$ & 5.60 \\
\hline
\end{tabular}

Nice traffic:

$$
N=10, \quad f=0.01, \quad E A=1 / 5, \quad E P=1 / 3, \quad E T=3.7500 .
$$


Table 3. Analytical results nice traffic

\begin{tabular}{rcccc}
\hline$R$ & $\rho$ & $\begin{array}{c}E B \\
\text { (packets) }\end{array}$ & $\begin{array}{c}E D \\
\text { (seconds) }\end{array}$ & $\begin{array}{c}\text { CPU-time } \\
\text { (seconds) }\end{array}$ \\
\hline 4 & 0.9375 & 93.1235 & 0.2433 & 4.48 \\
5 & 0.7500 & 9.7367 & 0.0210 & 4.16 \\
6 & 0.6250 & 4.5649 & 0.0072 & 4.33 \\
7 & 0.5357 & 3.8452 & 0.0053 & 5.56 \\
8 & 0.4688 & 3.7576 & 0.0050 & 8.02 \\
9 & 0.4167 & 3.7503 & 0.0050 & 12.10 \\
10 & 0.3750 & 3.7500 & 0.0050 & - \\
\hline
\end{tabular}

Table 4. Simulation results nice traffic

\begin{tabular}{ccccr}
\hline$R$ & $\begin{array}{c}95 \% \text { Confidence interval } \\
\text { for } E B\end{array}$ & $\begin{array}{c}\text { Interval } \\
\text { width }\end{array}$ & $\begin{array}{c}\text { Simulation time } \\
\text { (frames) }\end{array}$ & $\begin{array}{c}\text { CPU-time } \\
\text { (seconds) }\end{array}$ \\
\hline 4 & $91.4859-95.9760$ & 4.4900 & $16 \cdot 10^{6}$ & 239.93 \\
5 & $9.7176-10.1615$ & 0.4439 & $16 \cdot 10^{5}$ & 26.00 \\
6 & $4.5323-4.6622$ & 0.1299 & $4 \cdot 10^{5}$ & 6.26 \\
7 & $3.8125-3.8749$ & 0.0624 & $3 \cdot 10^{5}$ & 4.58 \\
9 & $3.7351-3.7810$ & 0.0458 & $3 \cdot 10^{5}$ & 4.37 \\
9 & $3.7325-3.7814$ & 0.0489 & $3 \cdot 10^{5}$ & 4.39 \\
\hline
\end{tabular}

\section{NUMERICAL ASPECTS}

Our first conclusion from the results is that the analytical results are inside the confidence intervals except the case $R=3$ for one way voice traffic. In this section we shall first discuss the three conditions mentioned in Sections 2 and 4 that have to be satisfied to solve the model. Secondly we will consider possible numerical bottlenecks in the algorithm. Thirdly we discuss the introduction of a checkpoint into the program and finally the results of this checkpoint are evaluated.

The first condition mentioned in Section 2 was $E T<R$. Since we knew $E T$ it was trivial to choose $R$ so that $E T<R$ was satisfied. The second condition was det $L \neq 0$, where $L$ is a square matrix defined in Section 4. Since this condition was satisfied in all our examples we will make the following conjecture:

\section{Conjecture 1: $\operatorname{det} L \neq 0$ for almost all $P$ and $R$}

By "for almost all $P$ and $R$ " we mean that if we choose the parameters specifying $P$ and $R$ randomly, then the set on which the statement is not true has probability zero.

In step 2 of the algorithm the zeros of $\operatorname{det}\left[z^{R} I-P A(z)\right]$ on the punctured unit disk are computed. In all our applications all these zeros had multiplicity 1 . Although it is not necessary for the solution of the model, we will also make the following conjecture:

Conjecture 2: all zeros of $\operatorname{det}\left[z^{R} I-P A(z)\right]$ on the punctured unit disk have multiplicity 1 for almost all $P, R$ and $N$

In step 4 of the algorithm a set of simultaneous linear equations must be solved. This was possible in all our examples. Hence:

Conjecture 3: the set of simultaneous linear equations constructed in step 3 of Section 2 is independent for almost all $P, R$ and $N$

The first numerical bottleneck in the algorith is the computation of $\operatorname{det}\left[z^{R} I-P A(z)\right]$. For small values of $p^{(1)}$ and $p^{(2)}$ a number of small probabilities have to be multiplied, which already causes inaccuracy in $\operatorname{det}\left[z^{R} I-P A(z)\right]$. Although the choice of a nice parameter set increases the possibilities of obtaining results for larger $N$, a matrix of transition probabilities like

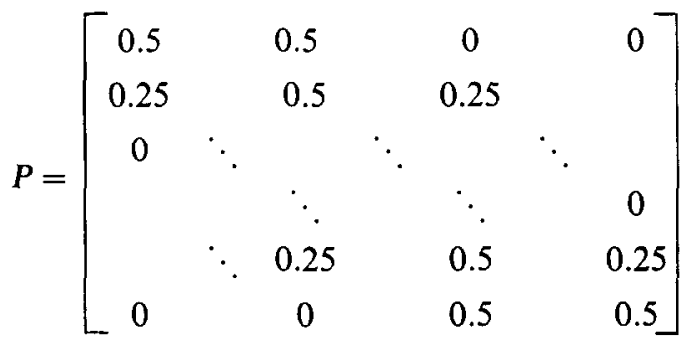


would be expected to increase the possibilities even further, because the non-zero probabilities are well-balanced and not so small. This was indeed confirmed by numerical experience.

The second numerical bottleneck is the computation of the zeros of $\operatorname{det}\left[z^{R} I-P A(z)\right]$. First the zero $z=1$ was divided out of $\operatorname{det}\left[z^{R} I-P A(z)\right]$ and the other zeros were determined with an accuracy of at least $10^{-12}$. This did not cause any problems. For small values of $p^{(1)}$ and $p^{(2)}$ the zeros on $0<|z|<1$ appeared to lie close to $|z|=1$. This is not surprising because in this case $\left[z^{R} I-P A(z)\right] \approx$ $\left[z^{R} I-A(z)\right]$ and all zeros of $\operatorname{det}\left[z^{R} I-A(z)\right]$ not equal to $z=0$ lie on $|z|=1$.

The third and final bottleneck is the solution of the set of simultaneous linear equations. This set was scaled first and the solution with an accuracy of at least $10^{12}$ did not cause any problems.

Now let us indicate a way of checking the accuracy of the obtained solution. In case of a tridiagonal matrix $P$ it is particularly easy to solve the set of equations. We determine $J_{0}(z)$ after calculation of column 0 of $\left[z^{R} I-P A(z)\right]^{-1}$. From (5) it is easy to find an iterative scheme from which $J(z)$ follows. However, this scheme does not employ the equation obtained from the last column of $\left[z^{R} I-P A(z)\right]$. We substituted $J(z)$ in this equation to check the obtained solution.

It appeared that mainly at high loads the computation became inaccurate. This explains why the case $R=3$ for one way voice traffic is outside the confidence interval. This confirms the fact that the first bottleneck is the most important one, because $R=3$ has the smallest number of zeros on $0<|z|<1$ and the smallest number of equations.

\section{EVALUATION AND CONCLUSIONS}

In this paper we considered the numerical solution of a model of a slotted transmission system. An algorithm was described and implemented on a computer. Stochastic simulation was performed to validate the results. We described a traffic model and chose two parameter sets. It appeared that a realistic parameter choice imposes rather severe restrictions on the applicability of the solution procedure. This was caused by assuming sources that vary on a time scale of seconds feeding a transmission system operating at a time scale of milliseconds. This difference in time scales causes an ill-balanced transition probability matrix $P$ introducing numerical instability into the computations.

From the results and the numerical aspects the main conclusions are:

1. Application is restricted to limited values of $N$, because of numerical inaccuracies in the calculation of the determinant of an ill-balanced $(N+1) \times(N+1)$ matrix.

2. When both $E A \gg f$ and $E P \gg f$ then the implemented algorithm is inaccurate especially at high loads.

3. The conditions 1, 2 and 3 needed to solve the model were satisfied in all examples.

Some additional conclusions are:

4. The program can be used in practice because the relative inaccuracies are small.

5. At high loads, the analytical algorithm is very much faster than simulation.

From conclusions 1 and 2 we can derive a direction for future research. We would like to investigate larger values of $N$. However in our applications it is not uncommon that $E A \gg f$ and $E P \gg f$. In other words, the source activity process and the transmission process occur on different time scales. In our model these processes were integrated at the same level. It is certainly worth trying to exploit this difference in time scales to justify assumptions for a model in which these time scales are distinguished explicitly. This model could be checked by the analytical and simulation program developed in this paper.

Acknowledgements-The author wishes to thank E. A. van Doorn and a referee for valuable suggestions during the preparation of the paper.

\section{REFERENCES}

1. B. van Arem, Discrete time analysis of a slotted transmission system: theoretical solution. Memorandum 624 , Univ. of Twente, The Netherlands (1987).

2. B. van Arem, Discrete time analysis of a slotted transmission system: numerical solution. Memorandum 625 , Univ. of Twente, The Netherlands (1987). 
3. H. Bruneel, Some remarks on discrete-time buffers with correlated arrivals. Comput. Opns Res. 12, 445-458 (1985).

4. J. N. Daigle and J. D. Langford, Queueing analysis of a packet voice communication system. IEEE Infocom '85, Proc., pp. $18-26$.

5. K. Sriram, P. K. Varshney and J. G. Shanthikumar, Discrete time analysis of integrated voice/data multiplexers with and without speech activity detcetors. IEEE J. Sel. Areas Commun. SAC-1, 1124-1132 (1983).

6. D. Anick, D. Mitra and M. M. Sondhi, Stochastic theory of a data-handling system with multiple sources. Bell Syst. Tech. J. 61, 1871-1894 (1982).

7. D. P. Gaver and J. P. Lehoczky, Channels that cooperatively service a data stream and voice messages. IEEE Trans. Commun. COM-30, 1153-1162 (1982).

8. A. Leon-Garcia, R. H. Kwong and G. F. Williams, Performance evaluation methods for an integrated voice/data link. IEEE Trans. Commun. COM-30, 1848-1857 (1982).

9. E. A. van Doorn, A. A. Jagers and J. S. J. de Wit, A fluid reservoir regulated by a birth-death process. Commun. Statist. Stochast. Models 4 (1988).

10. G. J. K. Regterschot, Wiener-Hopf factorization techniques in queueing models. Ph.D. thesis, Univ. of Twente, The Netherlands (1987)

11. M. A. Crane and D. L. Iglehart, Simulating stable stochastic systems III: Regenerative processes and discrete-event simulations. Opns. Res. 23, 33-45 (1975).

12. M. A. Crane and D. L. Iglehart, Simulating stable stochastic systems II: Markov chains. J. Assoc. Comput. Mach. 21 , 114-123 (1974).

13. P. T. Brady, A statistical analysis of on-off patterns in 16 conversations. Bell Syst. Tech. J. 47, 73-91 (1968). 